\title{
La critique génétique et les sciences cognitives
}

\author{
Jean-Louis Lebrave
}

\section{(2) OpenEdition}

Journals

Édition électronique

URL : https://journals.openedition.org/genesis/129

DOl : 10.4000/genesis.129

ISSN : 2268-1590

\section{Éditeur :}

Presses universitaires de Paris Sorbonne (PUPS), Société internationale de génétique artistique littéraire et scientifique (SIGALES)

\section{Édition imprimée}

Date de publication : 20 juin 2010

Pagination : 131-150

ISBN : 978-2-84050-697-3

ISSN : 1167-5101

\section{Référence électronique}

Jean-Louis Lebrave, «La critique génétique et les sciences cognitives », Genesis [En ligne], 30 | 2010, mis en ligne le 14 septembre 2012, consulté le 30 mars 2023. URL : http://journals.openedition.org/ genesis/129; DOI : https://doi.org/10.4000/genesis.129 


\title{
La critique génétique et les sciences cognitives
}

\author{
Jean-Louis Lebrave \\ Examinez le schéma de principe d'une grande machine mathématique [...] \\ Retournez le schéma en tous sens, vous ne découvrirez aucune boîte marquée : invention, réflexion, pensée créatrice. \\ Léon BRILLOUIN ${ }^{1}$ \\ En un mot, c'est le faire qui devenait apparent, au détriment du rendu. \\ La peinture s' exhibait comme un acte du peintre. \\ Jean-Joseph Goux ${ }^{2}$
}

L'idée d'explorer les rapports de la critique génétique et des sciences cognitives, et d'utiliser celles-ci pour enrichir celle-là, n'est pas nouvelle : dès 1979, André Lentin m'avait suggéré de participer au premier colloque de l'Association pour la recherche cognitive (ARC) qui venait de naître afin d'y présenter la problématique des processus d'écriture et de l'exploitation génétique des brouillons. Les sciences cognitives existaient depuis plus de vingt ans, mais elles étaient encore une idée neuve en France en 1979, du moins pour un jeune chercheur en sciences humaines. Pour ce qu'on pouvait deviner du sens du terme « cognitif » lorsqu'on n'était spécialiste ni d'intelligence artificielle, ni de neurosciences, ni de linguistique chomskyenne dans son noyau le plus « dur », on pouvait considérer que la cognition n'était rien de plus qu'un terme « moderne » pour désigner le fonctionnement de l'esprit. Or depuis sa naissance, la critique génétique aspirait à reconstituer les processus de production qui sont à l'origine de la création textuelle. Pour atteindre cet objectif, il paraissait aller de soi qu'on devait s'efforcer d'accéder à ce qui s'est « réellement » passé dans l'esprit du créateur. La présence d'informaticiens et de psychologues au sein de la toute jeune communauté des « cogniticiens » français confortait cet espoir. Le recours à l'informatique en sciences humaines était alors le symbole de l'accès de celles-ci au statut de disciplines scientifiques au sens fort, et faisait bon ménage avec une autre manifestation évidente de la rupture épistémologique qui marquait la naissance de la critique génétique, je veux parler du remplacement de la question du pourquoi - «Pourquoi écrivezvous ? » aura été la question posée aux écrivains du Xxe siècle depuis la fameuse enquête lancée par Littérature en 1919 - par la question du comment : « Comment écrit-on ${ }^{3}$ ? »

Après tout, les sciences cognitives ne faisaient-elles autre chose que prendre le relais sous une forme modernisée des réflexions et des explorations inaugurées par les écrivains eux-mêmes ${ }^{4}$

1. Vie, matière et observation, Paris, Albin Michel, 1959, p. 72.

2. Accrochages. Conflits du visuel, Paris, Des Femmes-Antoinette Fouque, 2007, p. 152.

3. C'est bien sûr le titre de ma propre communication à ce colloque. Voir Jean-Louis Lebrave, « Comment écrit-on ? », dans Domaine et objectifs de la recherche cognitive, Orsay, Association pour la recherche cognitive, 1982, p. 87-108.

4. Pour ne mentionner que les principaux : Edgar A. Poe, «The Philosophy of Composition», Graham's Magazine, vol. XXVIII, n 4, avril 1846, p. 163-167 ; Paul Valéry, Cours de Poétique. CEuvres, Paris, Gallimard, coll. « Bibliothèque de la Pléiade », t. I ; Writers at work : The Paris Review Interviews, New York, Viking, vol. I, 1957; vol. II, 1963 ; vol. III, 1967 ; vol. IV, 1976 ; vol. V, 1981 ; vol. VI, 1984 ; vol. VII, 1986 ; vol. VIII, 1988 ; vol. IX, 1992 ; Jean-Louis de Rambures, Comment travaillent les écrivains, Paris, Flammarion, 1992. 
avant d'être reprises par la critique génétique ? À l'évidence, les sciences cognitives en devenir constituaient un environnement propice pour l'exploration du fonctionnement de l'esprit du créateur au travail telle qu'elle était pratiquée depuis ses origines par la critique génétique.

Trente ans après le colloque fondateur de 1979, la rupture fondamentale initiée par les sciences cognitives devrait être plus aisément perceptible, notamment du fait des développements spectaculaires de notre connaissance du cerveau. C'est bien d'ailleurs à quoi invitent les déclarations enthousiastes des promoteurs des sciences cognitives. Deux exemples me serviront de point de départ pour le bilan critique que je me propose d'esquisser ici. L'un est caractéristique de la position des chercheurs en intelligence artificielle. C'est l'ouvrage collectif, intitulé Création et cognition, que Mario Borillo et Jean-Pierre Goulette ont consacré récemment aux apports des sciences cognitives dans l'exploration «scientifique » des processus de conception chez les architectes, les designers et les artistes 5 . L'autre illustre les thèses du représentant français le plus connu des neurosciences cognitives. C'est l'ouvrage $D u$ vrai, du beau, du bien, dans lequel Jean-Pierre Changeux vient de rassembler et d'unifier l'ensemble de ses cours au Collège de France sur ce qu'il a nommé lui-même la neuroesthétique 6 . Après un rappel de certaines des thèses défendues par ces deux ouvrages, je commenterai deux notions susceptibles de jouer un rôle décisif dans une approche cognitive des processus de création : le darwinisme mental d'une part, l'abduction et la résolution de problème d'autre part. J'évoquerai ensuite des recherches récentes en imagerie cérébrale sur la production et la compréhension de récits. L'intérêt de ces recherches pour la question qui m'occupe n'est pas seulement d'examiner l'apport des neurosciences quant à « ce qui s'est passé dans [l'esprit de l'écrivain] qui commande à la main et trace les mots sur un support ${ }^{7}$ ». On verra aussi que ces travaux d'imagerie se fondent sur les acquis de la recherche menée sur la production écrite en psychologie cognitive, seul secteur des sciences cognitives dont la convergence avec la critique génétique soit notable et ait commencé à donner des fruits. Je terminerai en replaçant la critique génétique dans le paysage intellectuel global qui a vu l'émergence des sciences cognitives après la Seconde Guerre mondiale.

\section{L'informatique, les neurosciences, la création}

Dans son introduction ${ }^{8}$, Mario Borillo affirme que les sciences de la cognition renouvellent profondément les rapports entre les « sciences formelles et sciences de la nature » et ce qu'il nomme la «sphère de la création », et il identifie trois axes pour cette rencontre d'un nouveau type. Le premier relève de ce qu'il appelle « l'art électronique », et concerne le rôle des technologies informatiques dans la production contemporaine d'œuvres d'art. Le deuxième concerne l'utilisation de l'informatique "dans la constitution, la gestion et l'utilisation des archives de l'art ». Enfin, le troisième est constitué par les perspectives nouvelles que

\footnotetext{
5. Mario Borillo et Jean-Pierre Goulette (dir.), Cognition et création. Explorations cognitives des processus de conception, Sprimont (Belgique), Mardaga, 2002.

6. Jean-Pierre Changeux, Du vrai, du beau, du bien. Une nouvelle approche neuronale, Paris, Odile Jacob, 2008.

7. C'est la formule employée par Michel Contat dans un article paru dans Le Monde du vendredi 17 novembre 1989. Il concluait d'ailleurs sur un constat désabusé : «Les neurosciences ont des choses à nous dire à ce sujet, mais rien, hélas, de décisif. Entre le cerveau et la main qui trace, la route de la connaissance, actuellement, est coupée. » 8. Mario Borillo, «Une rencontre symptomatique de la mutation computationnelle de notre temps, Art contemporain et Sciences de la cognition », dir. Mario Borillo et Jean-Pierre Goulette, Cognition et création, op. cit., p. 15-31.
} 
les sciences de la cognition introduisent dans la réflexion « sur la nature des processus de création par lesquels s'élabore [l'œuvre d'art]».

D'une manière plus incisive et polémique dans la forme, mais analogue quant à l'affirmation d'un changement de paradigme affectant les sciences humaines, Jean-Pierre Changeux place cette déclaration liminaire en tête du résumé de son cours de 2005 au Collège de France :

Le cours de l'année 2005 a été consacré à la poursuite des réflexions sur la neuroesthétique et tout particulièrement la relation entre peinture et musique. Depuis Aristote, on sait que l'homme est un animal raisonnable et social, doué de langage, qui développe une culture et possède une histoire. On sait également que les trois principaux champs de ses activités culturelles se regroupent autour de l'activité scientifique, de la régulation éthique et de la création artistique. [...]

En guise d'introduction, plusieurs présupposés idéologiques, qui sont monnaie courante dans les sciences de l'homme, ont été déconstruits. Première opposition réductrice : la dualité corps-esprit. Le programme de la neuroscience contemporaine est en fait d'abolir cette distinction archaïque, fondée sur une ignorance délibérée des progrès de la connaissance scientifique. [...] Deuxième opposition : l'opposition nature-culture. [...] Dernière opposition : le spirituel et le matériel ${ }^{9}$.

Ces déclarations triomphalistes sont de bon augure concernant l'apport des sciences cognitives à la connaissance des processus de création. Quel bilan peut-on tirer réellement en 2010 ?

\section{Des « sciences formelles »...}

L'ouvrage collectif dirigé par Mario Borillo et Jean-Pierre Goulette appelle une première remarque. En plaçant dans son introduction le renouvellement produit par les sciences cognitives sous la bannière des « sciences formelles » et des disciplines issues de la logique, Mario Borillo fait de la computation le cœur des sciences de la cognition. On est donc au cœur de ce que Léon Brillouin appelle les «machines mathématiques » dans la citation placée en exergue de cet article, et par laquelle je veux rappeler l'enracinement historique des sciences cognitives dans la cybernétique et la théorie de l'information. Par rapport à la « cognition spontanée » qui a entouré les premières réflexions sur la production littéraire, et même par rapport à la problématique cognitive que j'avais pu développer moi-même au début des années quatre-vingt-dix, il y a là une volonté de mettre en avant une rupture épistémologique fondamentale liée à la « révolution cognitive». Il ne s'agit pas d'un point secondaire, même s'il a été fréquemment occulté dans les débats sur l'apport des « sciences formelles » au développement des sciences de l'homme.

C'est bien l'ancrage dans la computation qui permet de comprendre pourquoi Mario Borillo revendique l'appartenance aux sciences cognitives des trois axes qu'il identifie, alors qu'on pourrait penser que cette attribution concerne seulement le dernier, consacré explicitement à la nature des processus de création, et ne vaut pas des deux premiers, qui relèvent plus directement du domaine des sciences et technologies de l'information.

Pour ce qui concerne la création littéraire, le premier de ces axes - l'art électronique pourrait correspondre à deux domaines. On peut penser d'abord à la génération automatique de textes pratiquée par Jean-Pierre Balpe. Mais celui-ci, en remplaçant le sujet écrivant par un logiciel d'écriture, a développé une démarche qui n'entretient pratiquement aucun

9. Repris - mais en tête du cours de 2004 - dans Du vrai, du beau, du bien, op. cit., p. 104-105. 
rapport avec la critique génétique telle qu'elle s'est constituée depuis trente ans. Un autre rapprochement pourrait être envisagé avec les mutations des processus de création littéraire induites par l'apparition du traitement de texte comme outil d'écriture. On rencontre ici plusieurs dimensions qui relèvent des sciences cognitives. Outre le problème des usages des nouveaux outils, dont l'évaluation pour la production littéraire reste à faire, de nombreuses questions relatives au fonctionnement de l'esprit dans un cadre technologique nouveau se posent. Quelles sont les relations entre la mémoire externe constituée par le manuscrit et, par exemple, la mémoire de travail et la mémoire à long terme ? Quels sont les effets du remplacement de l'espace graphique calibré et bien délimité du manuscrit par l'espace mobile et constamment variable de l'écran d'ordinateur ? Comment apprécier les bouleversements cognitifs induits par la frappe des lettres au clavier ? Le rapport intime du scripteur à sa propre écriture est atténué ou disparaît ; le flux scriptural continu du manuscrit est remplacé par un enchaînement discret de lettres distinctes ; des mouvements de frappe effectués par les deux mains sur les touches d'un clavier se substituent aux gestes d'écriture accomplis par une seule main sur le papier. Certaines de ces questions commencent à être abordées par la psychologie cognitive : étude des mouvements oculaires pendant la production d'écrits manuscrits, incidence des contraintes spatiales sur la production textuelle et sur le texte produit, et, symétriquement, étude des données fournies par l'enregistrement online des données de l'écriture au clavier ${ }^{10}$. Mais une exploration systématique, pourtant indispensable depuis la généralisation des outils d'écriture informatisés, n'a pas encore été menée à bien ${ }^{11}$.

En ce qui concerne le deuxième axe identifié par Mario Borillo, la constitution des archives de la création littéraire, leur gestion et leur utilisation constituent un volet majeur du programme de la critique génétique, et celle-ci a mené une réflexion poussée sur l'apport de l'informatique dans les deux domaines de l'édition électronique et de l'hypertexte12. Là aussi, le potentiel cognitif de ces questions est considérable, mais il n'a pas été réexaminé en tant que tel depuis que le développement d'Internet a pratiquement fait disparaître la problématique de l'hypertexte. Pourtant, et pour prendre un seul exemple, les théoriciens américains de l'hypertexte ont souligné dans les années quatre-vingt-dix la parenté entre le fonctionnement de la pensée et le caractère non linéaire, non séquentiel et non hiérarchique de l'hypertexte13, et parallèlement la critique génétique a utilisé l'hypertexte comme un outil théorique pour penser la genèse et représenter les manuscrits. S'agit-il d'une simple analogie ?

10. Voir Denis Alamargot, David Chesnet, Christophe Dansac et Christine Ros, «Eye and Pen : a new device to study reading during writing », Behavior Research Methods, Instruments and Computers, 38 (2), 2006, p. 287-299; Thierry Olive, Jean-Michel Passerault, Jean-Louis Lebrave et Nathalie Le Bigot, « La dimension visuo-spatiale de la production de textes : approches de psychologie cognitive et de critique génétique », Langages, 2010 ; Sven Strömqvist, "Une approche expérimentale du processus d'écriture : l'enregistrement de la frappe au clavier », Genesis, $\mathrm{n}^{\circ}$ 27, 2006, p. 45-58.

11. Voir néanmoins, pour une première approche, la table ronde «Écriture et informatique » organisée par le Centre de recherches linguistiques de l'université Paris X-Nanterre en mai 1989, et l'ouvrage coédité avec Jacques Anis : Jacques Anis et Jean-Louis Lebrave (dir.), Texte et ordinateur : les mutations du lire-écrire, deuxième édition augmentée, Paris, CRL, Paris X-Nanterre, 1993.

12. Voir par exemple Jean-Louis Lebrave, «Hypertextes - Mémoires - Écriture », Genesis, n 5, 1994, p. 9-24.

13. Voir par exemple Jay D. Bolter, Writing space. The Computer, Hypertext, and the History of Writing, Hillsdale, N. J., Lawrence Erlbaum Associates, 1991 ; P. Delany et George P. Landow (dir.), Hypermedia and Literary Studies, Cambridge, Mass., MIT Press, 1991. 
Historiquement, l'invention du concept d'hypertexte par Vannevar Bush est contemporaine de la naissance de l'informatique et des premiers développements de la cybernétique, qui sont elles-mêmes au fondement des sciences cognitives... La question mériterait donc d'être revisitée.

L'exploration du troisième axe par les collaborateurs de Mario Borillo et Jean-Pierre Goulette fait apparaître de nombreuses convergences avec la théorie développée par la critique génétique. Par exemple, Philippe Boudon écrit à propos de la conception architecturale :

[...] on ne peut comprendre l'espace architectural en tant qu'il a été conçu en se contentant d'《 analyser » cet espace architectural, [...] un déplacement est nécessaire vers ce que, en architecturologie, nous appelons l'« espace de conception », que je définirai ici comme une fiction théorique postulée d'un espace qui est celui dans lequel se déroulent les opérations de l'architecte au travail ${ }^{14}$.

De même, à propos du rôle du croquis dans le design, Nigel Cross évoque la nécessité d'un " processus de conception », « au cours duquel on atteint peu à peu, et parfois péniblement, les dessins définitifs, à travers une série d'autres dessins appelés croquis », ceux-ci permettant « l'exploration simultanée du problème et de la solution 15 ».

Ces phrases trouveraient aisément leur place dans n'importe quel ouvrage de critique génétique, et on pourrait multiplier les exemples. Cette observation ne fait d'ailleurs que rejoindre un constat déjà ancien : pour une part, le vocabulaire de la critique génétique est commun aux écrivains et aux peintres et aux sculpteurs. Comme l'a rappelé Pierre-Marc de Biasi,

c'est [...] le langage des peintres et des sculpteurs qui a souvent fourni aux écrivains leur métadiscours professionnel [...] aux peintres, on a emprunté les termes de «croquis », « esquisse », « ébauche, « réserve », « touche », « retouche », « repentir », « travail sur le motif », « non finito », etc. ; aux sculpteurs, « pioche », « dégrossissage », « polissage », « modèle », « gabarit », «moule ${ }^{16} \ldots$

On est dès lors amené à se poser deux types de question. D’abord, quel est le rapport véritable des développements de Philippe Boudon ou Nigel Cross avec les sciences cognitives, et plus particulièrement les « sciences formelles »? Ou, symétriquement, la critique génétique auraitelle pratiqué les sciences cognitives sans le savoir ? Ces deux questions peuvent se ramener à une seule : la rupture évoquée en introduction est-elle moins marquée qu'il n'y paraît ?

\section{... au darwinisme mental}

Les suggestions de Jean-Pierre Changeux pour le domaine de la peinture appellent des remarques du même ordre.

14. Philippe Boudon, «Éléments pour une "simulation" scientifique des "opérations" de conception (ou peut-on rendre "analysable" une compétence majeure de l'architecte, la conception », M. Borillo et J.-P. Goulette, Cognition et création, op. cit., p. 52.

15. Nigel Cross, « Comprendre la pensée du concepteur », ibid., p. 42-44.

16. Pierre-Marc de Biasi, « Génétique des arts », dans Olga Anokhina et Sabine Pétillon (dir.), Critique génétique.

Concepts, méthodes, outils, Paris, IMEC éditeur, coll. « Inventaires », 2009, p. 179. 
La thèse générale défendue par Changeux sur le plan des neurosciences est connue : c'est celle du darwinisme mental que le neurobiologiste a développée depuis L'Homme neuronal pour rendre compte de l'extraordinaire plasticité du fonctionnement cérébral et en particulier de sa maturation après la naissance. L'application du darwinisme mental au domaine de l'art est présentée dans son dernier livre17 à partir du célèbre texte de Poe sur la " philosophie de la composition » dont la critique génétique a pour sa part fait dès ses débuts abondamment usage. On le sait, Poe souligne « combien serait intéressant un article écrit par un auteur qui voudrait, c'est-à-dire qui pourrait raconter, pas à pas, la marche progressive qu'a suivie une quelconque de ses compositions pour arriver au terme définitif de son accomplissement». Pourquoi Changeux se place-t-il sous l'autorité de Poe pour affirmer que «les premières étapes du processus créateur » sont une «expérience mentale » « au caractère darwinien évident »? Un rappel du texte utilisé par Changeux nous aidera à comprendre. Je le cite ici dans la traduction de Baudelaire :

[...] les laborieux et indécis embryons de pensée, la vraie décision prise au dernier moment, l'idée si souvent entrevue comme dans un éclair et refusant si longtemps de se laisser voir en pleine lumière, la pensée pleinement mûrie et rejetée de désespoir comme étant d'une nature intraitable, le choix prudent et les rebuts, les douloureuses ratures et les interpolations.

Par rapport à l'original anglais, on peut qualifier la traduction de Baudelaire de belle infidèle : le poète accentue la dramatisation (présente néanmoins dans l'original à travers l'évocation de tout ce qui se passe « derrière la scène ») en mettant en place une scénographie à suspense dans laquelle la création s'accomplit dans la douleur. Si Poe nous éloigne du mythe romantique de l'inspiration, Baudelaire tend à remplacer celle-ci par une théâtralisation au fond tout aussi romantique, et en tout cas tout aussi éloignée de l'activité réelle de production de l'écrivain. Ainsi par exemple des « innumerable glimpses of idea that arrived not at the maturity of full view », que Baudelaire rend par " l'idée si souvent entrevue comme dans un éclair et refusant si longtemps de se laisser voir en pleine lumière ». Changeux n'indique pas la provenance de la traduction qu'il utilise. Plus fidèle dans sa littéralité que celle de Baudelaire (ainsi, Changeux transcrit l'exemple précédent par « les innombrables éclairs d'idées qui n'atteignent point la maturité de la pleine lumière »), elle n'est pas neutre pour autant : prenant un parti inverse de celui de Baudelaire, Changeux élimine tout ce qui concerne la théâtralité, en particulier tout le passage, qui suit immédiatement les lignes qu'il cite, dans lequel Poe évoque

les rouages et les chaînes, les trucs pour les changements de décor, les échelles et les trappes - les plumes de coq, le rouge, les mouches et tout le maquillage qui, dans quatre-vingt-dix-neuf cas sur cent, constituent l'apanage et le naturel de l'histrion littéraire.

Et Changeux choisit de mettre particulièrement en lumière les « essais et erreurs » qui sont au fondement de son interprétation néodarwinienne de la création artistique. Dans les tâtonnements évoqués par Poe, il retient donc de manière préférentielle la multiplicité des possibles, ce foisonnement qui a pour origine l'équivalent de ce que Changeux appelle ailleurs un « moteur de diversité »- autrement dit une instance analogue aux algorithmes

17. Du vrai, du beau, du bien, op. cit., p. 142-147. Mais une note précise qu'il s'agit d'une « partie d'un texte datant de $1987 »$. 
par lesquels les informaticiens engendrent des suites de nombres pris au hasard ${ }^{18}$. Quant au processus par lequel une solution est choisie parmi tous ces « essais », il est l'équivalent de la mise en œuvre de mécanismes adaptatifs.

On doit saluer le caractère très novateur du darwinisme mental que Jean-Pierre Changeux propose pour rendre compte de la maturation du système nerveux et de la " sélection naturelle » qui s'opère au sein des liaisons synaptiques au cours de cette maturation. Cette théorie ouvre notamment une troisième voie très prometteuse dans le débat entre l'inné et l'acquis. Mais la pertinence réelle de son exportation dans le domaine de la création artistique laisse sceptique. Sans doute, les ratures, les remplacements, les alternatives non résolues, les pistes esquissées puis abandonnées peuvent-ils être considérés comme des essais au sein desquels ce qui est rejeté ou remplacé peut prendre rétrospectivement le statut d'une erreur. Mais par-delà cette analogie somme toute superficielle, plusieurs différences majeures opposent la création littéraire au modèle darwinien. Dans la conception darwinienne de l'évolution, des mutations strictement aléatoires sont sélectionnées de manière mécanique par l'environnement dans lequel elles ont lieu : c'est l'association du hasard et de la nécessité dont Jacques Monod a fait le titre de son livre. Or le processus de création littéraire contredit ces principes essentiels du darwinisme. D'abord, on ne peut assimiler l'instance qui écrit ni à un moteur de diversité aléatoire ni à un mécanisme de sélection contextuelle automatique. L'apparition du verbe vouloir dès le début de l'Ébauche de La Terre de Zola (« Je veux faire le poème de la terre »), l'excitation que la «vacherie » d'Hérodias fait naître chez Flaubert (« la vacherie d'Hérodias pour Saint Jean-Baptiste m'excite ») sont autant de traces visibles d'un sujet doté d'intentions, de désirs, de vouloirs. C'est lui qui opère la sélection des éléments finalement retenus, et non un simple effet contextuel aveugle. Ensuite, il est difficile de définir de manière opératoire un contexte qui provoquerait mécaniquement la sélection : le contexte est instable et reste partiellement indéterminé jusqu'à l'achèvement de l'œuvre, notamment parce que jusqu'à celui-ci, il est constamment modifié par ce que la cybernétique appelait une boucle de rétroaction (la « sortie » du système vient réalimenter l'« entrée »). Enfin, on ne peut parler de nécessité que de manière rétrospective, en adoptant le point de vue de l'œuvre achevée. Placer le processus lui-même sous le signe de la nécessité procéderait d'une conception purement téléologique qui est aux antipodes du darwinisme19, mais qui va aussi à l'encontre des principes posés par la critique génétique dès ses débuts. On pourrait encore ajouter que l'évolution des espèces est toujours et à jamais en cours. Au contraire, l'évolution du processus de création s'arrête avec la mort du créateur. Par contre, la publication de l'œuvre marque le début d'un autre processus évolutif, celui de la réception, qui s'apparente beaucoup plus à l'évolution darwinienne des espèces que le processus de

18. La dimension probabiliste du darwinisme est évidente. Voir par exemple la remarque suivante du philosophe François Guery : « [...] il est vrai que la méthode darwinienne, probabiliste, ne prend le vivant que comme une masse, puisque la population ne se transforme qu'en vertu d'une "loi des grands nombres" qui rend plus ou moins probables la ou les variations favorables », François Guery, «Le darwinisme social », Le Magazine littéraire, $\mathrm{n}^{\circ} 176$, 1985.

19. De plus, comme l'a fait remarquer Daniel Ferrer, si Poe avait réellement suivi le cheminement qu'il décrit dans ce texte fameux, on en viendrait « à se demander comment [il] a pu jamais écrire autre chose que The Raven ». Voir Daniel Ferrer, «La critique génétique : "Philosophy of Composition" ou "Gold Bug” ? », Études anglaises, n 53-3, juillet-septembre 2000, p. 284. 
production. La réception est l'affaire de la philologie, dont l'épanouissement est d'ailleurs contemporain de la diffusion des théories darwiniennes.

Dès lors, même si l'extension du darwinisme mental au domaine de la création peut constituer une métaphore commode pour mettre de l'ordre dans le foisonnement des traces des manuscrits, et sauf à prendre la notion dans un sens trivial - l'écrivain essaie, soupèse les variantes, choisit celles qui lui paraissent les plus adéquates à son projet - elle ne fait guère progresser notre connaissance effective du processus de création littéraire.

En revanche, les progrès réalisés grâce aux neurosciences dans la connaissance effective des mécanismes neuronaux à l'origine de notre perception des couleurs et des sons, et par là même des soubassements cérébraux de la création picturale et musicale sont incontestables. Je renvoie par exemple aux pages dans lesquelles Jean-Pierre Changeux explique au profane comment l'information visuelle est traitée par le cerveau, depuis les neurones de la rétine jusqu'au cortex visuel, puis comment on retrouve dans la pratique des peintres le fonctionnement différencié des aires spécialisées correspondantes ${ }^{20}$. De même, Oliver Sacks, en compilant toutes les données issues aussi bien de l'imagerie cérébrale que de la pathologie, montre de manière spectaculaire la diversité et la richesse des «implantations » cérébrales de la musique 21.

En conclusion de ce rapide survol, on peut s'étonner de la disparité entre l'optimisme des déclarations générales sur le changement de paradigme induit par les sciences cognitives et le caractère contestable des principes que je viens de présenter à propos de Mario Borillo et de Jean-Pierre Changeux - en tout cas, de leur faible efficacité si on tente de les appliquer à la création littéraire.

\section{Deux outils de modélisation cognitive}

Après avoir esquissé une analyse de la méthodologie générale, je m'attacherai maintenant à deux outils de modélisation cognitive fréquemment utilisés aussi bien par les collaborateurs de l'ouvrage de Mario Borillo et Jean-Pierre Goulette que par les chercheurs en psychologie cognitive : l'abduction et la résolution de problème.

\section{Des chiens, des grenouilles, du curare...}

Dans son acception contemporaine, la notion logique d'abduction a été élaborée par Peirce et constitue un élément central de sa sémiotique. Sous cette forme, elle est couramment utilisée dans le contexte du paradigme indiciaire de Carlo Ginzburg22, mais aussi de ce qu'on nomme en anglais depuis Horace Walpole la serendipity. Je ne l'aborderai pas ici sous cet angle général et me limiterai à l'aspect - très particulier - de son utilisation pour appréhender l'invention et la capacité de création dans l'épistémologie des sciences et dans les réflexions sur l'activité de conception des designers et des architectes. Elle est mentionnée

20. Jean-Pierre Changeux, Du vrai, du beau, du bien, op. cit., p. 118-134.

21. Oliver Sacks, Musicophilia, Paris, Éditions du Seuil, 2009.

22. Pour une exploitation dans le cadre de l'interprétation des traces génétiques, voir Daniel Ferrer, «Le matériel et le virtuel : du paradigme indiciaire à la logique de mondes possibles », dans Michel Contat et Daniel Ferrer (dir.), Pourquoi la critique génétique?, Paris, CNRS Éditions, 1998, p. 11-30. 
par exemple par Nigel Cross, qui signale que la conception est abductive. De même, JeanGabriel Ganascia et Claude Debru l'invoquent pour modéliser la démarche expérimentale de Claude Bernard 23 . Ils écrivent par exemple :

Étant donné une théorie initiale, le savant doit construire une procédure expérimentale capable de tester (c'est-à-dire corroborer ou réfuter) cette théorie [...]. Les inférences impliquées par cette analyse sont à l'évidence des abductions, car l'objectif est d'expliquer les faits observés en modifiant la théorie 24 .

Clairement, l'abduction fonctionne comme un générateur d'hypothèses qui permet de transformer la théorie initiale lorsque les prédictions de celle-ci ne concordent pas avec les données observées. On trouve par exemple dans le célèbre Cahier rouge de Claude Bernard la liste suivante de «projets d'expériences » :

Expérience consistant à enlever la vésicule de fiel chez un chien.

Détruire par injection le pancréas et les glandes salivaires. Injecter l'oxyde de carbone ; s'il agit sur les tissus comme sur les globules du sang ce sera bon. Ou bien encore : injection de chlorure de calcium, de chlore. Injection de soufre en fleur. Soufrage du pancréas.

Les cellules épithéliales de l'intestin sont-elles dissoutes par la bile?

L'action de l'éponge de platine change-t-elle l'amidon en sucre?

Injection d'huile dans le poumon. Qu'en résulte-t-il pour la respiration ? Les oiseaux ont l'air de n'en pas souffrir du tout. Chez le lapin cela produit très vite l'acidité de l'urine. Pourquoi ?

Que devient le sucre dans ce cas ? Injection de corps gras diversement oxydables ${ }^{25} \ldots$

Deux remarques à ce propos. D'une part, on voit comment l'abduction joue le rôle d'un véritable moteur d'invention : " concevoir une expérience susceptible de valider ou d'invalider une théorie est une tâche très complexe qui demande de l'intuition, de l'habileté et de l'imagination 26 ». Comme l'écrit Christiane Chauviré lorsqu'elle analyse les recherches de Peirce en vue d' « optimiser la recherche scientifique », l'abduction produit « un flot d'hypothèses », « un flot chaotique » qui submerge le chercheur, et au sein duquel il est nécessaire d'organiser « une stratégie rationnelle » qui permettra de classer ces hypothèses et d'éliminer rapidement celles qui sont fausses ${ }^{27}$. Je retiendrai ici bien sûr non le caractère anarchique du foisonnement, mais la créativité de l'abduction, particulièrement manifeste dans le texte de Claude Bernard que je viens de citer. À propos de cet aspect de la pensée de Peirce, Christiane Chauviré parle d'ailleurs en se référant à Leibniz d'un véritable projet d'Ars inveniendi28. D'autre part, on voit qu'à la racine du raisonnement abductif, on trouve

23. Jean-Gabriel Ganascia et Claude Debru, « CYBERNARD : A Computational Reconstruction of Claude Bernard's Scientific Discoveries », dir. Lorenzo Magnani, Ping Li, Model-Based Reasoning in Science, Technology and Medicine, Berlin/New York, Springer Verlag, 2007, p. 497-510.

24. Ibid., p. 499-500.

25. Claude Bernard, Cahier de notes. 1850-1860, présenté et commenté par Mirko Drazen Grmek, Paris, Gallimard, 1965 , p. 71.

26. Jean-Gabriel Ganascia et Claude Debru, «CYBERnARD : A Computational Reconstruction of Claude Bernard's Scientific Discoveries », art. cit., p. 500.

27. Christiane Chauviré, «L'économie de la recherche selon Charles Sanders Peirce », Revue de métaphysique et de morale, $\mathrm{n}^{\circ} 47,2005 / 3$, p. 391-402.

28. Ibid., p. 402. 
l'étonnement de l'observateur, qui constate un phénomène qui ne répond pas à son attente. C'est cet étonnement qui est à l'origine de la mise en œuvre de l'abduction. On en trouve par exemple une illustration remarquable dans une page du Cahier $7 i 29$ de Claude Bernard que je voudrais commenter brièvement.

Dans ce cahier de laboratoire, Claude Bernard fait un compte rendu des résultats d'expériences conduites dans le cadre de son cours de 1849 au Collège de France. Il cherche à vérifier une hypothèse qui lui a été suggérée par des expériences sur l'absorption du curare par les muqueuses : «on n'absorberait les miasmes que par la muqueuse pulmonaire ». Dans ce but, il fait une première expérience dans laquelle il injecte une dissolution de curare dans la trachée d'un chien : «l'animal n'éprouva aucun mauvais effet de la substance bien que la solution ait baigné dans la muqueuse bronchique $30 »$. Il poursuit :

$2^{\circ}$ le lendemain je fais une injection sur le même

chien en poussant avec force la solution au moyen

d'une seringue dans le poumon. L'animal tousse

au moment de l'injection.

9 out 7 ou 8 minutes après le chien était mort

empoisonné des effets du curare

Pourquoi donc ce chien est-il mort le lendemain

et pas la veille?

Cela tient à ce que la veille le curare n'avait que touché la muqueuse trachéale et non celle des petites bronches.

Cette dernière muqueuse absorberait donc

le venin et la première ne l'absorberait pas ? C'est ce dont je me suis assuré par les expériences suivantes.

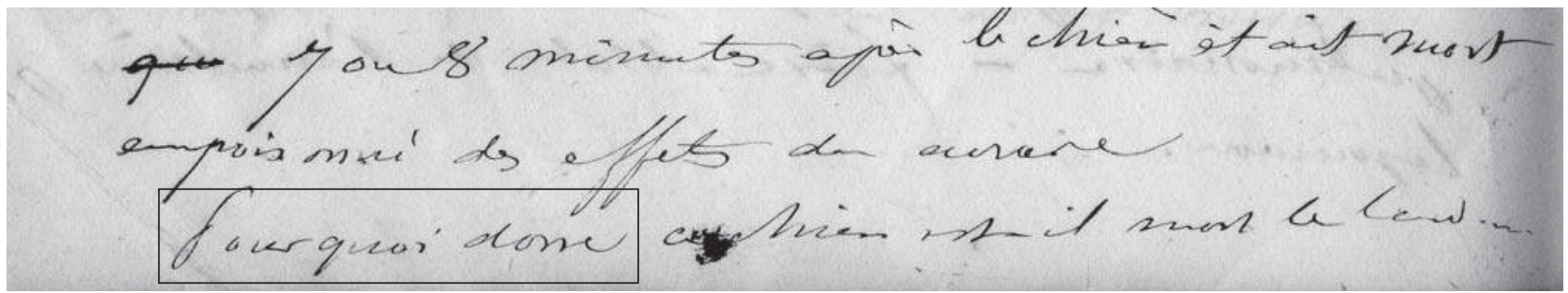

Claude Bernard, Cahier 7i, bas de la page 22

(c) Fonds Claude Bernard/

Collection Collège de France/

IMEC

29. Fonds Claude Bernard du Collège de France, Institut Mémoire de l'édition contemporaine.

30. Cahier 7i, p. 22. 


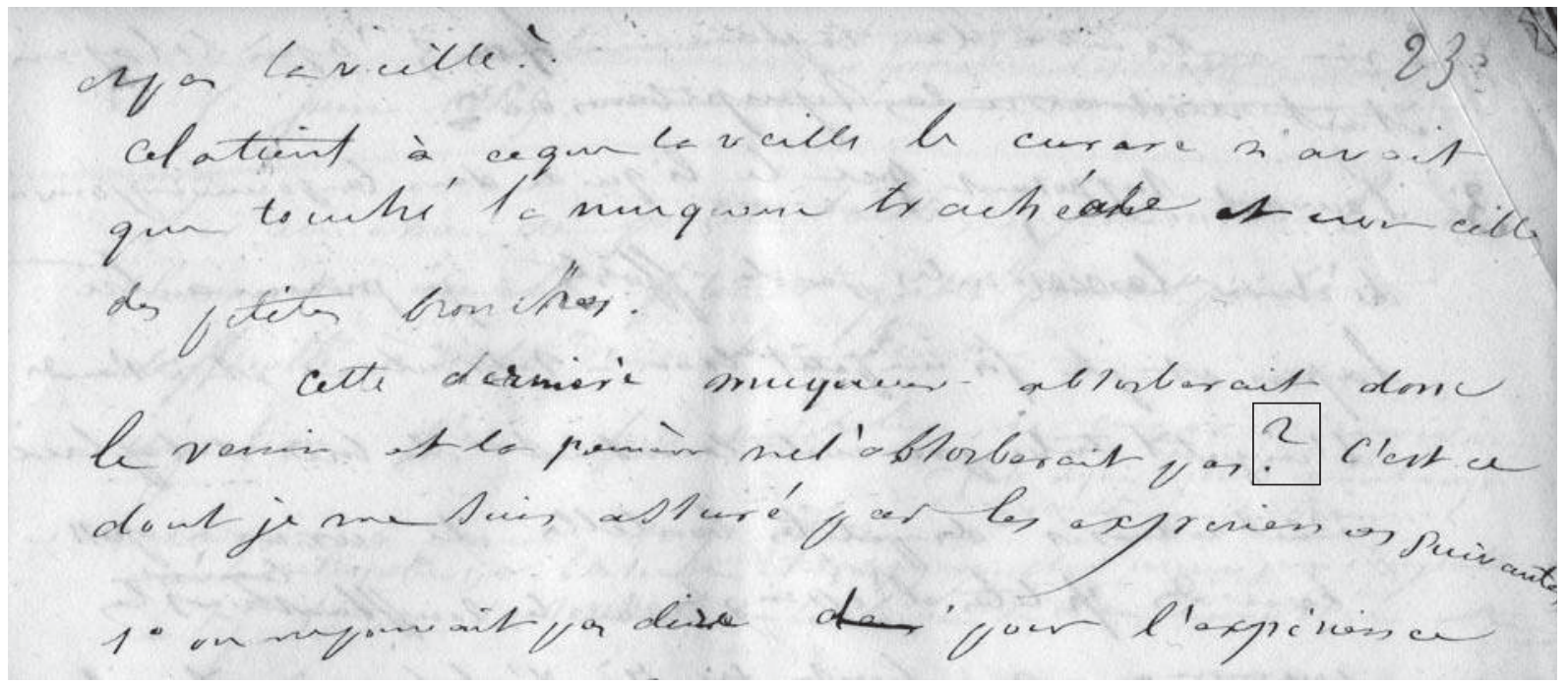

Claude Bernard relate alors trois expériences destinées à vérifier cette hypothèse. Ces expériences à leur tour suggèrent une nouvelle hypothèse et une nouvelle idée d'expérience : « prendre une boule pour prouver que la muqueuse trachéale n'absorbe pas [...] barbouiller haut de la page 23 (c) Fonds Claude Bernard/ avec un pinceau ».

On voit bien sur cet exemple l'extraordinaire fécondité de l'abduction chez Claude Bernard. Mais on est loin du mécanisme aveugle sous-jacent au darwinisme mental, et on est plus proche de l'instance dotée d'intentions, de désirs, et de vouloirs que j'ai évoquée plus haut à propos de la création littéraire. Il paraît pourtant difficile de transposer cet étonnement générateur d'abductions à l'activité créatrice de l'écrivain, sauf, comme c'est une fois encore le cas chez Zola, si l'univers fictionnel né du travail de création possède une telle prégnance que les enchaînements causaux peuvent y faire l'objet de raisonnements analogues à ceux de Claude Bernard sur les animaux de son laboratoire. L'abduction paraît pouvoir s'appliquer moins à l'invention littéraire qu'à la créativité du design, de la recherche et de la démarche scientifique et l'étonnement générateur d'abduction caractériser davantage le généticien, voire le lecteur ordinaire, que l'écrivain lui-même.

Enfin, on voit bien le « rendement » du recours à l'abduction dans le cadre d'une réflexion générale sur la logique et la modélisation. L'abduction fait système avec la déduction et l'induction en clôturant de manière élégante un paradigme d'opérations logiques qui est aussi un paradigme lexical. Et dans le cadre de la simulation du raisonnement humain par des procédures d'intelligence artificielle, l'abduction permet d'espérer implémenter une des formes de la créativité du savant sur un ordinateur. En revanche, on peut être un peu plus réservé quant à son apport pour la compréhension de l'invention elle-même, dans la mesure où l'abduction paraît constituer un moyen de filtrer l'invention proprement dite plutôt qu'une explication de la créativité de celle-ci. En outre, si la démarche scientifique consiste bien à construire des théories générales à partir de cas particuliers, et, comme chez Claude Bernard, à modifier ces lois pour intégrer une observation surprenante, il est douteux qu'on puisse 
appliquer ce constat à la création artistique : même Zola choisit de modifier la biographie de Gervaise plutôt que de modifier les lois de l'hérédité et du crime qu'il veut illustrer avec La Bête humaine.

\section{«Un drame violent à donner le cauchemar à tout Paris »}

Le couple problème-solution occupe une place centrale dans les réflexions du volume dirigé par Mario Borillo et Jean-Pierre Goulette, comme l'illustre par exemple la contribution dans laquelle Jean-Pierre Chupin discute différents modèles cognitifs de la conception architecturale (boîte noire, boîte de verre, « wicked problems », etc.) ${ }^{31}$. Or la résolution de problème (problem solving) joue un rôle majeur dans un grand nombre de modèles élaborés en intelligence artificielle et en psychologie cognitive - ce qui, dans ce dernier cas, implique également de nombreuses applications à la production verbale écrite dans le registre pédagogique de la didactique de l'écriture. On peut donc considérer qu'il s'agit d'un outil potentiellement important pour les recherches cognitives dans le champ de la critique génétique. Est-il fructueux d'étendre son utilisation au domaine de la création littéraire et, plus globalement, des processus de création en général ?

En montrant que les écrivains eux-mêmes envisagent l'œuvre encore à l'état de projet comme un désir à satisfaire ou une tâche à accomplir, les avant-textes de Zola ou de Flaubert suggèrent qu'il s'agit d'une piste intéressante. J'ai cité plus haut l'Ébauche de La Terre. De même, Zola commence l'Ébauche de La Bête humaine en écrivant :

Je voudrais, après Le Rêve, faire un roman tout autre ; d'abord dans le monde réel ; puis sans description, sans art visible, sans effort, écrit d'une plume plus courante ; du récit tout simplement ; et comme sujet, un drame violent à donner le cauchemar à tout Paris, quelque chose de pareil à Thérèse Raquin, avec un côté de mystère, d'au-delà, quelque chose qui ait l'air de sortir de la réalité (pas d'hypnotisme, mais une force inconnue, à arranger, à trouver). Le tout dans une grande passion évidemment. L'amour et l'argent mêlés. Mais surtout l'argent, voire la jalousie 32 .

Dans les deux cas, l'écrivain pose un objectif initial : faire le poème de la terre, écrire un drame violent qui donne le cauchemar à tout Paris, etc. Tout le processus dont le manuscrit de l'Ébauche conserve la trace consiste à affiner progressivement la définition de cet objectif et à élaborer les moyens d'y parvenir, ce qu'on peut aussi formuler comme la construction de solutions qui permettront d'atteindre l'objectif visé, c'est-à-dire de résoudre le problème initial 33 .

L'ensemble s'opère à travers l'enchaînement d'énoncés métalinguistiques remarquables. Après les travaux fondateurs d'Henri Mitterand qui les a commentés le premier, ils ont été étudiés notamment d'un point de vue linguistique par Almuth Grésillon, et dans une

\footnotetext{
31. Jean-Pierre Chupin, «"La mariée mise à nu..." (à propos de l'enseignabilité des modèles de la conception) », M. Borillo et J.-P. Goulette, Cognition et création, op. cit., p. 65-96.

32. Émile Zola, Ébauche de La Bête humaine, Bibliothèque nationale de France.

33. Voir aussi sur ce point les remarques de Daniel Ferrer dans « La toque de Clementis, rétroaction et rémanence dans les processus génétiques », Genesis, n 6, « Enjeux critiques », 1994, p. 93-106. En se référant à Michael Baxandall, il souligne notamment le fait que le problème «se redéfinit en permanence » et que c'est « chemin faisant » que se construit l'intention (p. 98).
} 
perspective de génétique contrastive par Daniel Ferrer 34 . Ce dernier parle à leur propos de « protocole opératoire », et de fait, très spectaculaire est la parenté des Ébauches de Zola avec les protocoles qui, dans les procédures expérimentales des psychologues, accompagnent la méthodologie du problem solving. Rappelons par exemple que dans leurs expériences, Linda Flower et John Hayes demandent au sujet de dire à haute voix « tout ce qu'il pense et tout ce qui lui vient à l'esprit » pendant qu'il résout le problème que constitue la tâche d'écriture 35 .

Peut-on généraliser ? On peut penser par exemple que la même heuristique de résolution de problème est sous-jacente à la démarche de Flaubert lorsque après avoir terminé Un cœur simple et fait le vide sur sa table de travail, " excité » par " la vacherie d'Hérodias pour Saint Jean Baptiste », il commence à prendre des notes et à construire des scénarios pour le dernier des Trois Contes. D'une manière plus indirecte, mais fondamentalement apparentée, on peut aller, comme le fait Daniel Ferrer, jusqu'à attribuer une dimension métadiscursive à tous les enchaînements de ratures et de réécritures des avant-textes, ce qui les inscrit dans la méthodologie du problem solving.

À première vue séduisante, cette généralisation est discutable, dans la mesure où, plutôt que d'affiner notre appréhension du processus de création, elle tend à diluer la spécificité de la création littéraire dans le vague d'un modèle passe-partout applicable à pratiquement n'importe quelle action. En relation avec les protocoles expérimentaux des psychologues, il serait plus intéressant d'explorer une autre piste qui m'est suggérée par une remarque d'Henri Mitterand à propos des Ébauches de Zola : il rappelle que la rhétorique telle qu'elle était encore pratiquée au XIX⿸e siècle était aussi « un enseignement de la composition narrative et théâtrale, reposant sur l'étude approfondie de la logique des situations et des effets ». Comme le souligne Henri Mitterand, le calcul des situations, des relations entre les personnages et des enchaînements narratifs dans les Ébauches s'inspire directement de ces préceptes issus de la rhetorica utens. Et Zola n'est certainement pas un cas isolé. De même qu'Henri Mitterand évoque Marmontel et ses Éléments de littérature à propos de Zola, on a pu chercher dans la «méthode de travail » (Arbeitsweise) de Heine la trace de l'enseignement de l'abbé Daulnoy au lycée français de Düsseldorf. Le jeune Heine a d'ailleurs lui-même emprunté une Poétique françoise de Marmontel à la bibliothèque de Düsseldorf ${ }^{36}$. Or, même si la chose n'est pratiquement jamais signalée, le modèle de Hayes et Flower est lui aussi profondément enraciné dans la tradition rhétorique, puisque la recherche d'idées, la réalisation du plan, la mise en langue et la révision ne sont pas différentes au fond de la séquence canonique enchaînant dans la rhétorique l'inventio, la dispositio, l'elocutio et le travail de la lime. Il y aurait une recherche à mener sur le ré-habillage en termes de « cognition » des anciennes heuristiques de composition

34. Voir par exemple Henri Mitterand, « Le méta-texte génétique dans les Ébauches de Zola », Genesis, $\mathrm{n}^{\circ} 6$, « Enjeux critiques », 1994, p. 47-60 ; Daniel Ferrer, « Combien d'enfants avait Lady Gervaise ? », dans Jean-Pierre Leduc-Adine (dir.), Zola, genèse de l' cuvre, Paris, CNRS Éditions, 2002, p. 17-32 ; Almuth Grésillon, «Langage de l'ébauche : parole intérieure extériorisée », Langages, ${ }^{\circ} 147$, septembre 2002, p. 19-28; Halina Suwala, Philippe Hamon et Jean-Pierre Leduc-Adine, « L'ébauche de L'Argent », dans Philippe Hamon et Jean-Pierre Leduc-Adine (dir.), Mimesis et Semiosis. Littérature et représentation, Paris, Nathan, 1992, p. 39-55.

35. Voir John R. Hayes et Linda Flower, «Writing as Problem Solving », Visible Language, vol. XIV, n 4, 1980, p. 388-399.

36. Voir Jean-Louis Lebrave, Le Jeu de l'énonciation en allemand d'après les variantes manuscrites des brouillons de H. Heine, thèse de doctorat d'État, université Paris-Sorbonne, 1987, p. 89-92. 
transmises par la tradition rhétorique depuis l'Antiquité - et sur l'oubli concomitant par les chercheurs contemporains en sciences cognitives de cet enracinement dans la rhétorique. Symétriquement, les travaux d'imagerie cérébrale que je vais évoquer maintenant suggèrent que ces universaux rhétoriques ont un correspondant dans le fonctionnement général du cerveau.

\section{S'habiller, se doucher, raconter}

Un certain nombre d'expériences ont été conduites en neurosciences pour identifier les aires du cerveau impliquées dans la compréhension et la production de récits. Un premier bilan en a été dressé en 2004 par le neuropsychologue canadien Raymond A. Mar. L'objectif de celui-ci était de tester la convergence entre les modèles élaborés en psychologie cognitive pour rendre compte de la production et de la compréhension des récits et les données fournies par l'imagerie cérébrale sur cette question ${ }^{37}$. Il est significatif qu'au moment de donner une définition du récit, Mar choisisse de renvoyer à Barthes et à l'article qui ouvre le numéro 8 de la revue Communications consacré à « l'analyse structurale du récit ${ }^{38}$ ». De fait, à la suite des formalistes russes et notamment de la diffusion des travaux de Propp sur les contes, l'analyse des récits a constitué un temps fort du structuralisme. Sans refaire ici l'historique d'un phénomène bien connu, rappelons seulement que c'est sur la base des travaux structuralistes fondateurs que la linguistique textuelle, puis la psycholinguistique ont développé une série de modèles qui font partie de l'inventaire dressé par Mar39.

Ce rappel débouche sur une première observation. La confrontation entre les données de l'imagerie et les modèles psycholinguistiques n'est possible qu'au prix d'une simplification de ces derniers, dont la résolution est jugée par Mar « trop fine » pour pouvoir être exploitée telle quelle par l'imagerie cérébrale, que les travaux portent sur des patients cérébrolésés ou sur des sujets sains. Or les modèles utilisés par la psychologie cognitive sont eux-mêmes le produit d'une réduction par rapport aux analyses de la linguistique textuelle. Il n'est donc guère surprenant que la première réaction du généticien des textes littéraires face aux données utilisées par les expériences d'imagerie soit le scepticisme, tant est large le fossé qui sépare l'un de l'autre les ingrédients de ces « récits » et l'univers romanesque qui lui est familier. Mar cite une étude menée par Sophie Crozier, Angela Sirigu et leurs collaborateurs dans laquelle les sujets doivent détecter des erreurs dans l'enchaînement d'une phrase (test « syntaxique ») et dans celui des actions d'un script. Les données de leur exemple sont «s'habiller/prendre une douche ». Les deux actions successives enchaînées de manière fautive sont donc « s'habiller », puis «prendre une douche ». La conception de ces expériences dérive bien de la définition structuraliste du récit que donne Mar - «description d'une série d'actions et

37. Raymond A. Mar, « The neuropsychology of narrative: story comprehension, story production and their interrelation », Neuropsychologia, $\mathrm{n}^{\circ} 42,2004$, p. 1414-1434. Raymond Mar anime par ailleurs un magazine en ligne sur la «psychologie de la fiction » : <www.onfiction.ca/ $>$.

38. Roland Barthes, «Introduction à l'analyse structurale des récits », Communications, n 8, 1966, p. 1-27.

39. Il faudrait citer toute la Textlinguistik allemande. Voir par exemple le volume collectif édité par Wolfgang Dressler en 1978 : Current Trends in Textlinguistics, Berlin/New York, De Gruyter, 1978, ou, dans une perspective plus proche de l'intelligence artificielle et de la psychologie cognitive, Robert de Beaugrande, Text, Discourse and Process. Towards a Multidisciplinary Science of Texts, Norwood, N.J., Ablex Publishing Corporation, 1980. 
d'événements qui se déroulent dans le temps en se conformant à des principes causaux 40 »-, mais l'usage de cette définition paraît à première vue grossier et simpliste ${ }^{41}$.

Mais ce jugement à son tour doit être tempéré par une seconde observation. Mutatis mutandis, les données utilisées dans les protocoles expérimentaux d'imagerie cérébrale ne sont pas aussi éloignées qu'il y parait de ce qu'on peut observer dans les avant-textes des grands corpus romanesques du XIXe siècle. Qu'on songe par exemple aux enchaînements d'hypothèses et de calculs auxquels se livre un Zola dans ses Ébauches. Dans l'Ébauche de La Bête humaine, il écrit par exemple : «Il faudrait avoir l'histoire, mettre d'abord l'histoire sur ses pieds » ( $\left.f^{\circ} 353\right)$. Et les avant-textes zoliens fourmillent de «calculs » narratifs qui sont réellement du même ordre que l'exemple utilisé par Sophie Crozier et Angela Sirigu. J'en donnerai un seul exemple. Au début du premier chapitre du «premier plan détaillé » de La Bête humaine, Zola met en place la scène inaugurale du roman. Roubaud, sous-chef au Havre, est un «employé modèle » dont le « seul défaut » est d'être républicain, ce qui lui vaut d'être « appelé à Paris pour recevoir une réprimande sérieuse, à cause d'un manque de politesse envers le sous-préfet du Havre ». Zola poursuit (p. 17-18 du manuscrit) :

\section{Donc}

par dépêche

Roubaud appelé à Paris pour son

savon (on le menace de le casser, il fait des

excuses écrites (?) Et, par dessous, on le pardonne, à cause de son excellent service.

Il a amené sa femme Severine, avec un billet de faveur demandé à Rouen (donc le temps de le demander, pas eon appelé par dépêche, lui laisser un temps)

On voit que Zola commence par noter que Roubaud est « appelé à Paris pour son savon ». Ultérieurement, il ajoute «par dépêche » dans l'interligne (cet ajout est toutefois nécessairement antérieur à la parenthèse qui l'annule, il doit donc être quasi immédiat). Lorsqu'il introduit le «billet de faveur », ce détail est incompatible avec l'appel par dépêche, et c'est à ce momentlà que Zola note la parenthèse « donc le temps de le demander, pas appelé par dépêche, lui laisser un temps ». On est bien dans la « description d'une série d'actions et d'événements qui se déroulent dans le temps en se conformant à des principes causaux » évoquée par Mar, et la contradiction entre l'obtention d'un billet de faveur et la convocation par dépêche est exactement du même ordre que celle qui interdit qu'on mette ses vêtements pour prendre une douche. On peut donc tout à fait supposer que les mêmes aires cérébrales ont été activées chez Zola lorsqu'il conçoit la parenthèse que chez les sujets non cérébrolésés auxquels Sophie Crozier et Angela Sirigu ont soumis leur test.

40. Raymond A. Mar, « The neuropsychology of narrative... », art. cit., p. 1415.

41. Une étude d'imagerie cérébrale plus récente utilise un matériel expérimental plus riche que celui de l'étude menée par Sophie Crozier et Angela Sirigu. Sans être encore équivalentes à celles des grands romans du XIXe siècle, ces données sont moins déroutantes pour un œil habitué aux dossiers génétiques de Flaubert ou de Zola. Voir Nicole K. Speer et al., « Reading Stories Activates Neural Representations of Visual and Motor Experiences », Psychological Science, vol. XX, Nr 8, 2009, p. 989-999. 
Dans cet exemple, il est patent que plusieurs tâches sont effectuées en parallèle : Zola continue à inventer les détails du plan qu'il est en train d'écrire pour la première scène de son roman, mais ce faisant, sa mémoire a visiblement gardé l'accès à l'ajout qu'il vient de faire de "par dépêche ». S'il est difficile de démêler ici production et compréhension (les deux processus se déroulent certainement de manière parallèle), il est certain que l'incompatibilité entre la dépêche et le billet de faveur est détectée presque instantanément. Ceci s'accorde avec les résultats expérimentaux décrits par Mar, qui montrent que les aires activées en production et en compréhension sont les mêmes. Si l'on tient compte en outre de l'existence de mécanismes comparables au niveau « syntaxique » (dans les mêmes expériences, on soumet aux sujets des phrases comportant une anomalie dans l'ordre des composants syntaxiques), il est tentant dès lors de prolonger l'extrapolation. On peut imaginer par exemple que ce seraient ces zones impliquées dans le contrôle de la cohérence et du bon enchaînement causal des constituants du récit et du discours qui permettraient la mise en place de la distance que la relecture du déjà écrit crée chez le scripteur. On aurait alors, au moins sous forme d'ébauche, la contrepartie cérébrale de la « double locution » dont j'ai montré l'importance dans le processus d'écriture chez Heine ${ }^{42}$. Ce sont certainement ces mécanismes de contrôle de la cohérence qui interviennent lorsqu'il insère à la relecture un commentaire métalinguistique sur l'inadéquation d'un terme qu'il a employé lors de l'écriture de premier jet et corrige l'expression initiale par une forme de rature linéarisée.

Ces pistes sont intéressantes. On pourrait les prolonger d'une manière beaucoup plus spéculative, par exemple en cherchant à mettre en relation l'analyse qu'Almuth Grésillon et moi avons menée sur les brouillons du début d'Hérodias ${ }^{43}$ avec des données neurologiques. On s'en souvient, ces manuscrits font voir de manière spectaculaire les difficultés qu'éprouve Flaubert à changer de point de vue dans la description de la citadelle de Machaerous : à partir du point de vue issu des lectures de Flaubert, qui est celui du voyageur contemplant avec effroi dans l'Antiquité la forteresse depuis le pied de la montagne, et en passant par le point de vue omniscient synthétique résumé dans le plan circulaire du folio 732 verso (la citadelle vue de dessus), où placer le narrateur décrivant la citadelle sans préjuger du point de vue « subjectif » d'Hérode Antipas ? Plusieurs brouillons très raturés et le recours à un croquis sommaire sont nécessaires à Flaubert pour parvenir à la phrase pourtant très simple : « Quatre vallées profondes l'entouraient, une en face, deux sur les flancs, la quatrième par-derrière. » Or des travaux menés en collaboration par Alain Berthoz ont montré le lien entre une pathologie particulière liée à la difficulté à changer de point de vue et des lésions de l'hémisphère gauche dans des aires « supportant des modules pariéto-frontaux impliqués dans la mémoire visuo-spatiale de travail, un système pariéto-temporal et des aires préfrontales 44 », ces mêmes lésions pouvant être à l'origine de troubles épileptiques. L'existence de l'épilepsie de Flaubert est loin de faire l'unanimité chez les critiques. Pourtant, dans une synthèse récente,

42. Jean-Louis Lebrave, « Le jeu de l'énonciation en allemand d'après les variantes manuscrites des brouillons de H. Heine », art. cit. Voir aussi Almuth Grésillon et Jean-Louis Lebrave, « Linguistique et génétique des textes : un décalogue », numéro spécial du Français moderne, 2008, p. 37-49.

43. Almuth Grésillon, Jean-Louis Lebrave et Catherine Fuchs, « Flaubert : "Ruminer Herodias". Du cognitif-visuel au verbal-textuel », dans Daniel Ferrer et Jean-Louis Lebrave (dir.), L'Écriture et ses doubles. Genèse et variation textuelle, Paris, CNRS Éditions, 1991, p. 27-109.

44. Alain Berthoz, Résumés des cours au Collège de France pour l'année 2007, p. 346. 
le neurologue Michel Weber attribue sans équivoque à l'épilepsie les symptômes décrits par Maxime Du Camp et par Flaubert dans sa correspondance 45 . Se référant à une étude d'Henri Gastaut, il considère que « l'origine occipitale et plus particulièrement au niveau de l'aire striée paraît indéniable, la lésion devant s'étendre en avant, vers le lobe temporal 46 ».

Les aires cérébrales responsables des problèmes neurologiques dont souffrait Flaubert paraissent correspondre partiellement à celles qui ont été identifiées chez les patients étudiés par Alain Berthoz. Dans les deux cas, on constate chez ces sujets une difficulté à changer de point de vue. S'agit-il d'un simple rapprochement anecdotique, ou cette coïncidence ouvre-t-elle une vraie piste de recherche ? L'étude de la mise en place des repérages spatiaux dans les avant-textes flaubertiens n'a pas été menée de manière systématique. Si la difficulté rencontrée dans la description de la citadelle de Machaerous n'est pas un cas isolé, il serait passionnant de pouvoir corréler d'une manière autre que spéculative des problèmes apparaissant au niveau de la textualisation et la maladie probable de Flaubert.

Au terme de ce parcours certainement trop partiel, le bilan de l'apport actuel des sciences cognitives à l'appréhension des processus de création artistique et scientifique apparaît nuancé.

En ce qui concerne les voies de la découverte scientifique, on commence à entrevoir la possibilité d'une modélisation, d'une simulation et d'une reconstruction de la démarche par laquelle le savant fait évoluer les théories scientifiques ${ }^{47}$. Avec les travaux menés en intelligence artificielle par Jean-Gabriel Ganascia et son équipe sur la méthode expérimentale de Claude Bernard, une forme d'expérimentation rétrospective in silico devient possible. On a vu par ailleurs qu'à travers la formalisation de l'abduction, ces cheminements cognitifs impliquent la figure d'un observateur, qui s'étonne de l'incompatibilité de données expérimentales avec la «théorie initiale » dont il dispose, et qui met en marche un moteur générateur d'hypothèses qu'il testera en faisant évoluer la théorie. Des processus analogues interviennent à l'évidence dans la démarche de la création architecturale et du design, où le concepteur doit résoudre un problème initial (construire un bâtiment répondant à des spécifications particulières, concevoir un objet satisfaisant à un cahier des charges précis, etc.). On a vu aussi avec l'exemple de Zola que des mécanismes comparables sont susceptibles d'intervenir dans la création littéraire (satisfaire un vouloir initial, créer un univers fictionnel dont les composantes ne soient pas en conflit les unes avec les autres, respecter les règles d'enchaînement chronologique et causal, etc.). Néanmoins, et malgré le caractère stimulant de ces recherches, la création en tant que telle paraît devoir excéder encore pour un certain temps ce type de modélisation.

Il est sans doute plus productif de rechercher ce qu'on pourrait appeler des « universaux cognitifs » susceptibles d'entrer comme composants dans le processus de création. Plusieurs candidats peuvent être envisagés. On pensera d'abord aux bases neurales de l'écriture en tant qu'activité graphomotrice. Le même caractère d'universalité appartient aux éléments
Vers des universaux cognitifs

45. «L'épilepsie de Gustave Flaubert », Épilepsies, vol. VIII, n 3, juillet-août-septembre 2006, p. 155-159.

46. Ibid., p. 156.

47. Voir en outre dans ce numéro l'entretien avec Claude Debru. 
constitutifs de l'écriture en tant que technologie cognitive impliquant des interactions entre un dispositif technologique et des processus cérébraux. De même, comme on l'a vu, les études d'imagerie cérébrale portant sur la production et la compréhension de récits suggèrent que celles-ci font appel à des « représentations sensorielles et motrices de base », comme l'écrivent Nicole Speer et al. en conclusion de leur article sur la lecture de récits 48 . Enfin, c'est à la recherche de tels universaux que se consacrent les recherches de psychologie cognitive sur l'écriture, et c'est dans ce domaine que les coopérations entre généticiens du texte et psychologues commencent à être les plus fructueuses. On a pu montrer ${ }^{49}$ par exemple la parenté entre la catégorisation des stratégies d'écriture en «classique » et « romantique » proposée par le psychologue David Galbraith et le couple « écriture à programme/écriture à processus » proposé par Louis Hay en 198450. Cette rencontre prometteuse n'est certainement que la première sur un chemin qui promet d'être long.

48. Nicole K. Speer et al., « Reading Stories Activates Neural Representations of Visual and Motor Experiences », art. cit., p. 997. Plus globalement, elles ajoutent en conclusion de leur étude : « the use of sensory and motor representations during story comprehension may reflect a more general neural mechanism for grounding cognition in real-world experiences » (ibid., p. 998).

49. Denis Alamargot et Jean-Louis Lebrave, « The Study of Professional Writing. A Joint Contribution from Cognitive Psychology and Genetic Criticism », European Psychologist, vol. XIV/4, 2009.

50. Louis Hay, « Die dritte Dimension der Literatur », Poetica, Amsterdam, 1984, col. 16, n 3-4. Repris dans Louis Hay, La Littérature des écrivains. Questions de critique génétique, Paris, José Corti, 2002, p. 71 sq. 
JEAN-LOUIS LEBRAVE est directeur de recherche émérite à l'ITEM (CNRS-ENS). Il a publié des travaux sur les manuscrits de Heine (1987), sur les manuscrits de Proust (Proust à la lettre, en collaboration avec A. Grésillon et C. Viollet, Du Lérot, 1990) et de Flaubert («Ruminer Hérodias », en collaboration avec A. Grésillon et C. Fuchs, dans D. Ferrer et J.-L. Lebrave, L'Écriture et ses doubles, CNRS Éditions, 1991) et sur la théorie génétique ( «a critique génétique : une nouvelle discipline ou un avatar de la philologie ? », Genesis, $\left.n^{\circ} 1,1992\right)$. Il s'est intéressé en outre à l'hypertexte (« Hypertextes - Mémoires - Écriture », Genesis, n 5, 1994) et aux traitements informatiques des données manuscrites. Il a édité avec J. Anis un ouvrage sur le texte et l'ordinateur (Texte et ordinateur : les mutations du lire-écrire, Espace européen, 1991). Par ailleurs, il s'est attaché à l'analyse des dimensions psycholinguistiques de la critique génétique, liées au processus de l'écriture littéraire («La critique génétique et l'étude des processus d'écriture littéraires », dans Production du langage, dir. M. Fayol, Hermès/Lavoisier, 2002 ; D. Alamargot et J.-L. Lebrave, « The Study of Professional Writing. A Joint Contribution from Cognitive Psychology and Genetic Criticism », European Psychologist, vol. XIV, 4, 2009).

Jean-Louis Lebrave, jean-louis@lebrave.name

\section{Résumés \\ La critique génétique et les sciences cognitives}

Cet article explore les rapports entre la critique génétique et les sciences cognitives. Qu'il s'agisse des « sciences formelles» des chercheurs en intelligence artificielle ou du « darwinisme mental » élaboré par Jean-Pierre Changeux, l'ambition affichée des sciences cognitives est d'introduire un changement de paradigme radical dans l'appréhension des processus de création. L'importance de ce bouleversement est évaluée ici d'une part à partir de deux outils de modélisation cognitive utilisés en intelligence artificielle, l'abduction - souvent considérée comme le moteur de la créativité dans les sciences - et la résolution de problème, d'autre part à partir des premières expériences d'imagerie cérébrale consacrées à la production de récits. Ces recherches sont stimulantes, et le bilan provisoire est loin d'être négatif. Néanmoins, c'est sans doute la recherche d'universaux cognitifs impliqués dans les processus de création qui paraît pour l'instant la plus prometteuse, notamment à travers les coopérations entre critique génétique et psychologie cognitive.

This article explores the relationship between genetic criticism and cognitive science. Whether it be the "formal science" of artificial intelligence researchers or the "mental Darwinism" elaborated by Jean-Pierre Changeux, the open ambition of cognitive science is to introduce a radical change of paradigm in the approach to creative processes. We will evaluate the importance of this upheaval by first proceeding from two cognitive modeling tools used in artificial intelligence: abduction, often considered the motor of creativity in science, and problem solving. Secondly, we will proceed from the first experiences in brain imagery devoted to the production of narratives. This research is stimulating, and the provisional assessment is far from negative. Nonetheless, it is probably the search for cognitive universals implied in the creative process that appears most promising today, in particular in the cooperation between genetic criticism and cognitive psychology.
Dieser Beitrag hinterfragt das Verhältnis zwischen „critique génétique" und Kognitionswissenschaft. $\mathrm{Ob}$ es dabei um die formell orientierten Forscher in künstlicher Intelligenz geht oder um den von Jean-Pierre Changeux entwickelten „Mentaldarwinismus", das gemeinsam verteidigte Ziel besteht darin, im Verständnis von Schaffensprozessen einen radikalen Paradigmenwechsel einzuführen. Die Bedeutung dieses Umbruchs wird hier von zwei Begriffen aus beleuchtet, die in der künstlichen Intelligenz der kognitiven Modellisierung dienen : nämlich dem der „Abduktion“ - in den Naturwissenschaften häufig als Motor der Kreativität betrachtet - und dem der „Problemlösung“. Andererseits erscheint der Umbruch im Licht der ersten Versuche, in denen Bilder des Gehirns den Schreibprozess wiedergeben. Diese Forschungsergebnisse sind stimulierend, sodass eine provisorische Bilanz keineswegs negativ ist. Dennoch erscheint die zur Zeit meistversprechende Richtung die Erforschung der kognitiven Universalien zu sein, die in Schaffensprozesse impliziert sind; dies gilt vor allem für die Kooperationsprogramme zwischen „critique génétique“ und kognitiver Psychologie.

Este artículo explora las relaciones entre la crítica genética y las ciencias cognitivas. Ya se trate tanto de las "ciencias formales" de los investigadores en inteligencia artificial como del "darwinismo mental" elaborado por Jean-Pierre Changeux, la ambición explícita de las ciencias cognitivas es introducir un cambio de paradigma radical en la aprehensión de los procesos de creación. La importancia de esta transformación se evalúa aquí, por una parte, a partir de dos instrumentos de modelización cognitiva utilizados en inteligencia artificial, la abducción -considerada a menudo como el motor de la creatividad en las ciencias- y la resolución de problemas, y por la otra, a partir de las primeras experiencias de imágenes cerebrales centradas en la producción de relatos. Estas investigaciones resultan estimulantes y el balance provisorio está lejos de ser negativo. No obstante, es sin duda el estudio de los universales cognitivos involucrados en los procesos de creación el que aparece por el momento como el más prometedor, en particular a través de la cooperación entre crítica genética y psicología cognitiva. 
$\mathbf{O}$ artigo explora as relações entre a crítica genética e as ciências cognitivas. Quer se trate das "ciências formais" dos investigadores em inteligência artificial ou do "darwinismo mental" elaborado por Jean-Pierre Changeux, a fito das ciências cognitivas é introduzir uma radical mudança de paradigma na apreensão dos processos de criação. A importância desta revolução é avaliada com dois instrumentos de modelização cognitiva utilizados em inteligência artificial, a abdução frequentemente considerada o motor da criatividade nas ciências - e a resolução de problema, por um lado, e por outro com as primeiras experiências de imagiologia cerebral consagradas à produção de narrativas. $\mathrm{O}$ balanço provisório destas estimulantes investigações está longe de ser negativo. No entanto, é sem dúvida a investigação dos universais cognitivos implicados nos processos de criação que parece de momento a mais prometedora, nomeadamente através das cooperações entre crítica genética e psicologia cognitiva.
I 1 saggio esplora i rapporti tra la critica genetica e le scienze cognitive. Sia che si parli delle "scienze formali" degli studiosi di intelligenza artificiale o di "darwinismo mentale" elaborato da Jean-Pierre Changeux, l'ambizione chiara delle scienze cognitive è quella di introdurre un nuovo modello metodologico nello studio del processo creativo. L'importanza di tale mutamento d'orizzonte è qui analizzata incoraggiando i due strumenti di modellizazione cognitiva utilizzati nelle ricerche sull'intelligenza artificiale: da un lato, l'abduzione, spesso considerata come il motore creativo delle scienze; e, dall'altro, la risoluzione di problemi, in base ai primi esperimenti di radiografie del cervello osservato al momento di una produzione narrativa. Queste ricerche si rivelano particolarmente stimolanti, e, ad un primo provvisorio bilancio, tutt'altro che insicure. Nondimeno, è la ricerca di universali cognitivi implicati nel processo creativo che, indubbiamente, sembra per il momento l'ambito più promettente, grazie in particolare alle correlazioni tra critica genetica e psicologia cognitiva. 